This item was submitted to Loughborough's Research Repository by the author.

Items in Figshare are protected by copyright, with all rights reserved, unless otherwise indicated.

\title{
Manufacture of chitosan microbeads using centrifugally driven flow of gel- forming solutions through a polymeric micronozzle
}

PLEASE CITE THE PUBLISHED VERSION

\section{PUBLISHER}

(C) Elsevier

\section{VERSION}

AM (Accepted Manuscript)

\section{LICENCE}

CC BY-NC-ND 4.0

\section{REPOSITORY RECORD}

Mark, Daniel, Stefan Haeberle, Roland Zengerle, Jens Ducree, and Goran T. Vladisavljevic. 2009.

"Manufacture of Chitosan Microbeads Using Centrifugally Driven Flow of Gel-forming Solutions Through a Polymeric Micronozzle". figshare. https://hdl.handle.net/2134/5001. 
This item was submitted to Loughborough's Institutional Repository (https://dspace.lboro.ac.uk/) by the author and is made available under the following Creative Commons Licence conditions.

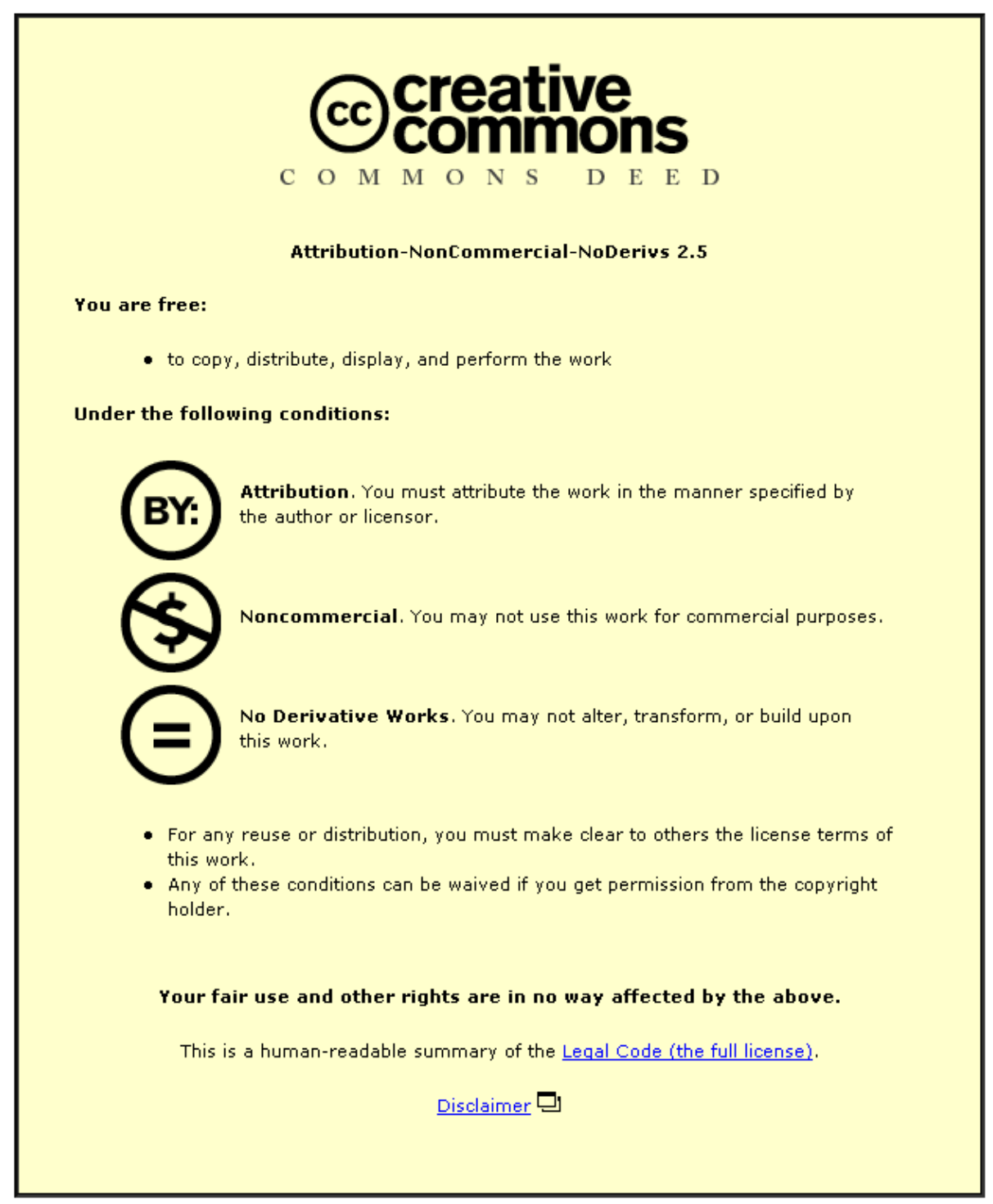

For the full text of this licence, please go to: http://creativecommons.org/licenses/by-nc-nd/2.5/ 


\title{
Manufacture of chitosan microbeads using centrifugally driven flow of gel- forming solutions through a polymeric micronozzle
}

Daniel Mark ${ }^{1}$, Stefan Haeberle ${ }^{1}$, Roland Zengerle ${ }^{1,2}$, Jens Ducree ${ }^{1,2}$ and Goran T. Vladisavljević ${ }^{3,4}$

${ }^{1}$ HSG-IMIT, Wilhelm-Schickard-Straße 10, D-78052 Villingen-Schwenningen, Germany

${ }^{2}$ Laboratory for MEMS Applications, Department of Microsystems Engineering (IMTEK), University of Freiburg, Georges-Koehler-Allee 106, D-79110 Freiburg, Germany

${ }^{3}$ Chemical Engineering Department, Loughborough University, Loughborough, Leicestershire LE11 3TU, UK

${ }^{4}$ Vinča Institute of Nuclear Sciences, PO Box 522, YU-11001 Belgrade, Serbia

\begin{abstract}
A centrifugally driven pulse-free flow has been used for generation of tripolyphosphate (TPP)-gelated chitosan beads with tunable diameters ranging from 148 to $257 \mu \mathrm{m}$. The production process requires a single motor as the sole actively actuated component. The $2 \%$ $(\mathrm{w} / \mathrm{w})$ chitosan solution was extruded through a polymeric nozzle with an inner diameter of $127 \mu \mathrm{m}$ in the centrifugal field ranging from 93 to $452 \mathrm{~g}$ and the drops were collected in an Eppendorf tube containing $10 \%(\mathrm{w} / \mathrm{w})$ TPP solution at $\mathrm{pH} 4.0$. The reproducibility of the bead diameters out of different nozzles was very good with overall CVs of the bead diameters down to $15 \%$ and the production rate was 45 beads per second per nozzle at $44 \mathrm{~Hz}$ rotor frequency. The production rate was proportional to the sixth power of the rotor frequency, which was explained by the non-Newtonian behaviour of the chitosan solution with a flow behaviour index of 0.466 . An analytical model for the bead diameter and production rate has been presented and validated by the experimental data. The shrinkage of chitosan drops during gelation was estimated from the observations and the theoretical model.
\end{abstract}


Keywords: hydrogel microbeads, chitosan, centrifugal, encapsulation, microfluidics, ionotropic gelation, monodisperse drops

Correspondence: Daniel Mark, HSG-IMIT-Institute for Micromachining and Information Technology, Wilhelm-Schickard-Straße 10, 78052 Villingen-

Schwenningen, Germany. Tel: +49 761203 7345. Fax: +49 761203 7322. E-mail: daniel.mark@hsg-imit.de

\section{Introduction}

Hydrogel microbeads made of biopolymers such as alginate, chitosan and gelatin find a wide range of applications in the food industry, agriculture, biomedicine and the pharmaceutical and cosmetic industry owing to their low toxicity, biocompatibility, biodegradability, high binding capacity to specific chemical species, and the ability to adsorb or release molecules in response to external signals or stimuli. They have been extensively used for encapsulation of enzymes and cells [1], in drug delivery [2], as scaffolds in tissue engineering [3], biosensors and actuators [4], sorbents [5], etc. The conventional methods for mass production of hydrogel microbeads are atomisation [6] and emulsification using conventional high-pressure valve homogenisers or rotor-stator systems [7]. The disadvantages of these techniques are a poor batch-to-batch consistency, poor control over the bead size and morphology and high degrees of bead polydispersity. A better control over the bead size can be achieved by extruding the gel-forming solution through a needle [8-9] or nozzle [10]. Detachment of drops from nozzle tip can be facilitated by vibrating the nozzle, providing a coaxial air or liquid 
flow or using an electrostatic potential to pull the droplets from a nozzle tip [11]. The major drawback of the extrusion techniques is a low production rate and the beads can be formed with tail threads, dents on the surface and a tear-like shape.

Over the past decade microengineering techniques for the production of hydrogel microbeads have been developed, such as micromolding [12], photolithography [13], microfluidic routes [14-16], microchannel emulsification [17] and membrane emulsification [18-19]. These processes are illustrated schematically in Figure 1 and listed in Table 1 along with the examples of the beads fabricated. The main benefit of using microengineering methods is the ability to precisely control the bead size, uniformity, shape and morphology. They can afford creation of complex 3D microstructures shown in Figure 2. Hollow beads have been produced by Kim et al. [26] using flow focusing glass microcapillary devices (Table 2). The Janus (bifacial) beads shown in Figure 2a) have been produced by Shepherd et al. [16] using a sheath-flow microfluidic device presented in Figure 1d). Square, hexagonal and cylindrical beads can be manufactured by micromolding or photolithography, whereas discoid beads can be fabricated by the gelation of deformed drops in shallow microfluidic channels with a high width-to-height ratio [29]. Micromolding and photolithography are relatively expensive techniques that require fabrication of photomasks or molds and are inconvenient for manufacturing spherical beads. Another disadvantages of photolithography include the need for photocrosslinkable materials and the possible detrimental effects of UV irradiation on encapsulants. Microfluidic routes shown in Fig. 1 are not suitable for production of gel beads by ionotropic external gelation, because the drop gelation at the channel outlet would lead to clogging of the channel. 
A three-dimensional gel network can be formed by chemical gelation (polymerisation by freeradical processes or via condensation) or by physical gelation, which can involve heating (heat-setting gels), cooling (cold-setting gels) or the addition of multivalent ions (ionotropic gelation). Calcium alginate beads are a typical example of the hydrogel particles generated by ionotropic gelation. The examples of novel microengineering techniques for production of alginate beads are shown in Figure 3. The same techniques can be used for fabrication of any hydrogel beads through the formation of salt bridges between charged macromolecules. As it is shown in Figure 4, the main strategies for initiating ionotropic gelation are internal gelation, external gelation, coalescence-induced gelation and rapid mixing. In the internal gelation [7], emulsified droplets contain a gel-forming polymer and a crosslinking agent in a nondissociated (inactive) form (e.g. $\mathrm{Ca}^{2+}$ in the form of $\mathrm{CaCO}_{3}$ ), whereas the continuous phase contains a species (e.g. $\mathrm{H}^{+}$) that diffuses into the droplets and triggers the release of the crosslinking agent and subsequent gelation. This method was used to generate Ca-alginate beads by diffusion of acetic acid from the continuous oil phase to the sodium alginate drops containing fine dispersion of $\mathrm{CaCO}_{3}[14,33]$. This approach will not be applied in this work because the resultant beads are often too soft and have a high tendency to aggregation, due to weak gelation [14]. In the external gelation, the droplets contain only a gel-forming biopolymer and a crosslinking agent diffuses from the external phase to induce gelation. It is usually a gelation mechanism in extrusion and atomisation processes, although it was recently used by Zang et al. [34] to produce Ca-alginate beads in flow focusing microfluidic device. Choi et al. [30] have fabricated Ca-alginate beads by direct rapid mixing of alginate and $\mathrm{CaCl}_{2}$ solutions in a microfluidic T-junction. Here, the drop formation rate should be greater than the gelation rate to suppress the gelation process before the drops are pinched-off from the junction. In the coalescence-induced process, the gel formation is achieved by coalescence of monodisperse droplets of a biopolymer solution with monodisperse droplets of a 
crosslinking solution [35]. However, it is impossible to completely prevent coalescence between the two sodium alginate drops, which leads to the formation of polydisperse beads when such bigger drop coalesces with the drop containing crosslinking solution.

In this work, chitosan gel microbeads will be fabricated by extruding chitosan solutions through a polymeric micronozzle using centrifugal force ranging from 93 to $452 \mathrm{~g}$ [36-37]. The ionotropic external gelation of chitosan drops will be achieved by crosslinking positively charged chitosan macromolecules with tripolyphosphate anions at $\mathrm{pH}$ below the $\mathrm{pK}$ value of chitosan, as can be seen in Figure 5. Chitosan is a linear polysaccharide composed of randomly distributed D-glucosamine and N-acetyl-D-glucosamine units linked by $\beta-(1-4)$ glycosidic bonds. It can be obtained by partial deacetylation of chitin extracted from the shells of crustaceans, such as shrimps, crabs, and lobsters. Chitosan beads have been widely used in drug delivery applications [38], for the immobilisation of cells, enzymes and antibodies [39], as chromatographic supports [40] and adsorbents for removal of metal ions from organic and aqueous solutions [41]. Conventional techniques for production of chitosan microbeads include spray drying [42], simple coacervation, complex coacervation with alginate [43], pressure-driven extrusion through nozzle [44] or needle [45], and emulsification combined with solvent evaporation and/or crosslinking with glutaraldehyde [46]. Recently, membrane emulsification [23] and flow focusing microfluidic devices [47] has been explored for the production of size controlled chitosan beads.

This work is the first experimental study of preparation of chitosan beads using micronozzle on a centrifugal platform. The advantages of this process are: i) The centrifugal force enables a pulse-free flow of gel-forming solution through micronozzle which allows for generation of uniformly sized beads. Size uniformity is essential in fundamental pharmaceutical and cell 
biological investigations, because it leads to enhanced control over drug release kinetics and precise control over the metabolism of encapsulated cells; ii) Several micronozzles can be operated simultaneously on the same platform; iii) The volume of gel-forming solution in the feed reservoir can be less than $500 \mu \mathrm{L}$, which is convenient for the development of new product formulations; (iv) The bead diameter can vary over a wide range and can precisely be controlled by adjusting the rotational speed of the centrifuge and the exit diameter of the nozzle; (v) An air gap between the nozzle outlet and the hardening solution prevents the nozzle from clogging, which is a clear advantage compared with microfluidic processes described in Figure 1; (vi) Unlike all fabrication methods shown in Figure 1 and 2, the beads are collected directly in standard Eppendorf tubes to allow easy post-production handling without cross contamination.

\section{Materials and methods}

Figure 6 illustrates the concept of bead production in this work and the experimental setup. The crosslinking solution is filled into Eppendorf tubes placed into holes in a swinging bucket rotor [37]. As soon as the centrifuge is started, the swinging buckets are driven into a horizontal position by the centrifugal pseudo-force $\boldsymbol{F}_{\omega}$ and the droplets that form at the tip of the micronozzles fly freely into the crosslinking solution and the gelation is initiated. The process can be monitored via a stationary stroboscopic camera and a flashlight (Fig. 7). The sub- $\mu$ s shutter speed allows sharp images even at the rotational frequencies of up to $100 \mathrm{~Hz}$. Figure 8 shows typical stages of the drop formation process captured by the stroboscopic camera. 
The size of the droplets that form at the nozzle can be determined by calculating the droplet diameter $d$ for which the gravitational (pseudo-)force surpasses the surface tension force. The gravitational force acting on the mass $m$ in a reference frame rotating with the frequency $v$ is

$$
F_{g}=m \omega^{2} r
$$

where $\omega=2 \pi v$ is the angular frequency and $r$ is the distance from the centre of rotation. The surface tension force $F_{\sigma}$ for a nozzle with diameter $d_{\mathrm{n}}$ is

$$
F_{\sigma}=\pi d_{n} \sigma
$$

where $\sigma$ is the surface tension of the liquid. For $F_{\mathrm{g}}=F_{\sigma}$ this leads to a droplet diameter of

$$
d=\sqrt[3]{\frac{6 d_{n} \sigma}{\rho \omega^{2} r}}
$$

with $\rho$ being the density of the liquid.

At the high peripheral speeds the air drag force $F_{\text {drag }}$ acting on the forming droplet has to be considered as well:

$$
F_{\text {drag }}=\frac{1}{2} C_{d} v^{2} \rho_{\text {air }} A
$$

where $C_{d}=0.1$ is the drag coefficient for a smooth sphere at $\operatorname{Re}>2 \times 10^{5}, v=\omega r$ is the peripheral velocity of the nozzle, $\rho_{\text {air }}=1.2 \mathrm{~kg} / \mathrm{m}^{3}$ is the density of the surrounding air and $A=\pi d^{2} / 4$ is the projected frontal area. At our maximum rotational frequency of $44 \mathrm{~Hz}, F_{\text {drag }}$ was less than $3 \%$ of the gravitational force and thus negligible in the following calculations.

In this study the gel forming biopolymer solution was 2 or $3 \%(\mathrm{w} / \mathrm{w})$ chitosan solution in $2 \%$ (w/w) acetic acid solution (medium molecular weight chitosan and glacial acetic acid, SigmaAldrich Chemie $\mathrm{GmbH}$, Germany). The beads produced with $1 \%$ (w/w) chitosan were very soft and prone to agglomeration. The crosslinking solution was a $10 \%(\mathrm{w} / \mathrm{w})$ sodium 
tripolyphosphate (TPP, 85 \%, Sigma-Aldrich Chemie GmbH, Germany) adjusted with acetic acid to $\mathrm{pH}$ 4.0. As a micronozzle we used a polyimide capillary with $127 \mu \mathrm{m}$ inner diameter (Detakta GmbH \& Co. KG, Germany). The particle size distribution of the resultant beads have been measured by a light scattering particle size analyser (LS 230, Beckman Coulter GmbH, Germany).

The beads have been produced simultaneously from two micronozzles and then the contents of the two Eppendorf tube were mixed together for measurements. Each nozzle was attached to a $300 \mu \mathrm{l}$ reservoir containing the chitosan solution. Drops have started to detach at about $20 \mathrm{~Hz}$ rotational frequency and the upper limit for safe operation of the rotor was $44 \mathrm{~Hz}$. The pressure difference $\Delta p\left(=2 \rho \pi^{2} v^{2} r^{2}\right)$ of the liquid in the nozzle should exceed the capillary pressure $p_{c}\left(=4 \sigma / d_{n}\right)$ before the liquid can flow through the nozzle. The minimum rotor frequency required for liquid flow can be calculated by solving the equation $\Delta p=p_{c}$ for $v$.

$v_{\min }=\frac{1}{\pi r} \sqrt{\frac{2 \sigma}{d_{n} \rho}}$

From Eq. [5] $v_{\min }=17 \mathrm{~Hz}$, which is in good correlation with the experimental observations.

\section{Results and discussion}

Figure 9 shows the variation of the bead diameter and the production rate with the rotor frequency for $2 \%(w / w)$ chitosan solution. The values of parameters in Eq. [3] used for plotting the theoretical curve shown with the solid line in Figure 9 (a) are: $d_{\mathrm{n}}=127 \mu \mathrm{m}$, $\sigma=63.4 \mathrm{mN} \mathrm{m}^{-1}$ (measured using the pending drop method with contact angle system OCA, DataPhysics Instruments GmbH, Germany), $\rho=1010 \mathrm{~kg} \mathrm{~m}^{-3}$, and $r=58 \mathrm{~mm}$. Table 3 shows the summary of the experimental results. The discrepancy between the drop diameters predicted 
by Eq. [3] and their measured values can be explained by shrinkage of the chitosan drops during gelation, because water is expelled from the drops as gelation progresses. The best fit line of Eq. [3] modified with a shrinkage factor $k_{\mathrm{s}}$ suggests that the bead diameter was reduced to about $69 \%$ of its original diameter during gelation. The drop diameters estimated by spot-checking stroboscopic images are in a good agreement with the diameters predicted using Eq. [3], as shown in Figure 9 (a).

The hardened beads have a relatively narrow size distribution with CVs between 15 and $22 \%$. Their size can be tuned between 148 and $257 \mu \mathrm{m}$ by controlling the rotational frequency. The effect of nozzle diameter on the particle size was not investigated in this work, but our previous work with alginate beads using the same technique $[36,37]$ shows that the size of microbeads is proportional to the third root of the nozzle diameter, $d_{n}$, as predicted by Eq. [3]. Particle size distribution histograms of the chitosan beads fabricated at 20 and $44 \mathrm{~Hz}$ with $2 \%$ (w/w) chitosan solution are shown in Figure 10, as well as three typical microscopic images of the beads. The initial production rate was 0.7 beads per second and nozzle at $20 \mathrm{~Hz}$ but increased greatly with the rotational frequency. At $44 \mathrm{~Hz}$ the bead production was still stable and more than 45 beads were produced per second and nozzle. The production rate could probably be increased further with a better rotor balance. A stable parallel production out of two nozzles demonstrate the suitability for parallelisation of the process on a single motor. The process can be further scaled up using four or six radially positioned nozzles with an additional advantage that gel beads of different chemical structure can be simultaneously fabricated on the same platform using different gel-forming solutions in different feed reservoirs. As shown in Figure 10 (d), the beads made from $3 \%$ chitosan solution were tearlike in shape, which could be attributed to faster gelation, as the rate of gelation was approximately proportional to the chitosan concentration. On the other hand, an increased 
viscosity of the chitosan solution leads to the decreasing tendency of the drops to regain its spherical shape, which is energetically the most favourable shape.

As Figure 9 suggests, the bead production rate $f_{\text {prod }}$ increases with approximately the $6^{\text {th }}$ power of the rotational frequency. To explain this tendency, we have to bear in mind that chitosan is a non-Newtonian, shear-thinning liquid [48]. The viscosity $\eta$ of a non-Newtonian liquid can be written as [49]:

$$
\eta=K \dot{\gamma}^{n-1}
$$

where $K$ is the consistency index, $\dot{\gamma}$ the shear rate and $n$ the flow behaviour index. HagenPoiseuille's law can be applied to predict the chitosan flow rate through the microcapillary assuming a laminar flow:

$$
Q=\frac{\Delta p}{\eta} \frac{\pi\left(d_{n} / 2\right)^{4}}{8 l}
$$

where $\Delta p$ is the pressure difference along the capillary of length $l$. Therefore, the mean shear rate is proportional to the volumetric flow rate $Q$ :

$$
\dot{\gamma}=\frac{u_{\max }}{d_{n} / 2}=\frac{\Delta p\left(d_{n} / 2\right)^{2}}{4 \eta l} \frac{1}{d_{n} / 2}=Q \frac{2}{\pi\left(d_{n} / 2\right)^{3}}
$$

where $u_{\max }$ is the velocity along the capillary axis. Substituting $\eta$ and $\dot{\gamma}$ from Eq. [6] using Eq. [7] and [8] respectively, we obtain

$$
C \frac{\Delta p}{Q}=Q^{n-1}
$$

where $C$ is a constant. To relate Eq. [9] to the bead production rate $f_{\text {prod }}$ we use the fact that the volume flow rate in the capillary is equal to the volume of a single drop multiplied by the number of drops generated per unit time:

$$
Q=V_{\text {drop }} f_{\text {prod }}=\frac{\pi d^{3}}{6} f_{\text {prod }} \stackrel{\text { (eq.3) }}{=} \frac{\pi}{6} \frac{6 d_{n} \sigma}{\rho \omega^{2} r} f_{\text {prod }}
$$


Considering that $\Delta p$ depends quadratically on the angular frequency $\omega$ and using Eq. [9] and [10], we obtain

$$
f_{\text {prod }}=a \omega^{\frac{2+2 n}{n}}
$$

where $a$ is a constant. Our experimental results shown in Figure 8 suggest a value of 6.29 for the exponent on $\omega$, resulting in a flow behaviour index of $n=0.466$ from Eq. [11]. This is consistent with the value of $n=0.497$ measured by Hwang and Shin [48] for a $2 \%$ chitosan solution at the shear rates greater than $10 \mathrm{~s}^{-1}$. In this work, the mean shear rate in the nozzle was in the range between 48 and $635 \mathrm{~s}^{-1}$, which corresponds to the apparent viscosity of the chitosan solution from 4.1 to $1.1 \mathrm{~Pa}$ s. 


\section{Conclusions}

We have developed a new microfluidic method for the cheap and reliable production of polyphosphate-gelated chitosan beads tunable in diameter between 148 and $257 \mu \mathrm{m}$, which permits their use for cell or drug encapsulation. The bead size decreased with increasing the rotational frequency and the ratio of the resultant bead diameter to the initial drop diameter was 0.69 , which was attributed to the drop shrinkage during the gelation process.

The production process requires a single motor as the sole actively actuated component. The potential for parallelisation was shown by producing beads simultaneously from two nozzles. The beads produced using $2 \%$ chitosan solution were spherical and very uniform in size with a coefficient of variation ranging between 15 and $22 \%$, proving very good reproducibility of sizes between different nozzles. The beads fabricated using $3 \%$ chitosan solution had tearlike shape due to faster gelation of drops and higher viscosity of the chitosan solution. The bead production rate was approximately proportional to the sixth power of the rotational frequency, which was explained by the non-Newtonian behaviour of the chitosan solution with a flow behaviour index of 0.466 . The rotor frequency was limited to $44 \mathrm{~Hz}$ which resulted in the maximum production rate of 45 beads per second per nozzle. With a wellbalanced rotor and multiple nozzles, the production rate could be significantly higher.

\section{Acknowledgements}

We would like to thank Hendrik Elsenheimer (IMTEK, Laboratory for Materials Process Technology) for providing help with the light scattering measurements. The stay of Dr Vladisavljević at IMTEK was sponsored by the Alexander von Humboldt Foundation (Bonn, Germany) under its follow-up sponsorship programme. 


\section{References}

[1] S. Fukui, A. Tanaka, Ann. Rev. Microbiol. 36 (1982) 145.

[2] P. Sriamornsak, J. Nunthanid, Int. J. Pharm. 160 (1998) 207.

[3] A. Khademhosseini, R. Langer, Biomaterials 28 (2007) 5087.

[4] T.C. Ayi, J.M.M. Tong, P.V.S. Lee, Proc. SPIE 6218 (2006) 62180K.

[5] G. Bayramoglua, A. Denizli, S. Bektas, M.Y. Arica, Microchem. J. 72 (2002) 63.

[6] J.C. Ogbonna, M. Matsumura, T. Yamagata, H. Sakuma, H. Kataoka, J. Ferment. Bioeng. 68 (1989) 40.

[7] D. Poncelet, R. Lencki, C. Beaulieu, J.P. Halle, R.J. Neufeid, A. Fournier, Appl. Microbiol. Biotechnol. 38 (1992) 39.

[8] T. Rehg, C. Dorger, P.C. Chau, Biotechnol. Lett. 8 (1986) 111.

[9] M.D. Fumi, A. Silva, G. Battistotti, O. Colagrande, Biotechnol. Lett. 14 (1992) 605.

[10] H.H. Lee, O.J. Park, J.M. Park, J.W. Yang, J . Chem. Tech. Biotechnol. 67 (1996) 255.

[11] C. Dulieu, D. Poncelet, R.J. Neufeld, In: W.M. Kühtreiber, R.P. Lanza, W.L. Chick (Eds.), Cell Encapsulation Technology and Therapeutics, Birkhäuser, Boston, 1999, p. 3.

[12] J. Yeh, Y. Ling, J.M. Karp, J. Gantz, A. Chandawarkar, G. Eng, J. Blumling III, R. Langer, A. Khademhosseini, Biomaterials 27 (2006) 5391.

[13] W.G. Koh, A. Revzin, M.V. Pishko, Langmuir 18 (2002) 2459.

[14] H. Zhang, E. Tumarkin, R.M.A. Sullan, G.C. Walker, E. Kumacheva, Macromol. Rapid Commun. 28 (2007) 527.

[15] A.S. Utada, E. Lorenceau, D.R. Link, P.D. Kaplan, H.A. Stone, D.A. Weitz, Science, 308 (2005) 537.

[16] R.F. Shepherd, J.C. Conrad, S.K. Rhodes, D.R. Link, M. Marquez, D.A. Weitz, J.A. Lewis, Langmuir, 22 (2006) 8618. 
[17] F. Ikkai, S. Iwamoto, E. Adachi, M. Nakajima, Colloid. Polym. Sci. 283 (2005) 1149.

[18] G.T. Vladisavljević, I. Kobayashi, M. Nakajima, R.A. Williams, M. Shimizu, T. Nakashima, J. Membr. Sci. 302 (2007) 243.

[19] T. Nakashima, M. Shimizu, M. Kukizaki, Adv. Drug Del. Rev. 6 (2000) 47.

[20] C. Qiu, M. Chen, H. Yan, H. Wu, Adv. Mater. 19 (2007) 1603.

[21] B.G. De Geest, J.P. Urbanski, T. Thorsen, J. Demeester, S.C. De Smedt, Langmuir, 21 (2005) 10275.

[22] S. Iwamoto, K. Nakagawa, S. Sugiura, M. Nakajima, AAPS PharmSci, article 25, 3 (2002) 1 .

[23] L.Y. Wang, G.H. Ma, Z.G. Su, J. Control. Release 106 (2005) 62.

[24] Q.Z. Zhou, L.Y. Wang, G.H. Ma, Z.G. Su, J. Colloid Interface Sci. 311 (2007) 118.

[25] J.W. Kim, A.S. Utada, A. Fernández-Nieves, Z. Hu, D.A. Weitz, Angew. Chem. Int. Ed. $46(2007) 1819$.

[26] J.W. Kim, A. Fernández-Nieves, N. Dan, A.S. Utada, M. Marquez, D.A. Weitz, Nano Lett. 7 (2007) 2876.

[27] E. Um, D.S. Lee, H.B. Pyo, J.K. Park, Microfluid. Nanofluid. 5 (2008) 541.

[28] J. Surh, G.T. Vladisavljević, S. Mun, D.J. McClements, J. Agric. Food Chem. 55 (2007) 175.

[29] I. Kobayashi, K. Uemura, M. Nakajima, Food Biophys. 3 (2008) 132.

[30] C.H. Choi, J.H. Jung, Y.W. Rhee, D.P. Kim, S.E. Shim, C.S. Lee, Biomed. Microdev. 9 (2007) 855 .

[31] S. Sugiura, T. Oda, Y. Aoyagi, R. Matsuo, T. Enomoto, K. Matsumoto, T. Nakamura, M. Satake, A. Ochiai, N. Ohkohchi, M. Nakajima, Biomed. Microdev. 9 (2007) 91.

[32] K.S. Huang, T.H. Lai, Y.C. Lin, Lab Chip 6 (2006) 954.

[33] X.D. Liu, D.C. Bao, W.M. Xue, Y. Xiong, W.T. Yu, X.J. Yu, X.J. Ma, Q.J. Yuan, Appl. 
Polym. Sci. 87 (2003) 848.

[34] H. Zhang, E. Tumarkin, R. Peerani, Z. Nie, R.M.A. Sullan, G.C. Walker, E. Kumacheva, J. Am. Chem. Soc. 128 (2006) 12205.

[35] S. Sugiura, T. Oda, Y. Izumida, Y. Aoyagi, M. Satake, A. Ochiali, N. Ohkohchi, M. Nakajima, Biomaterials 26 (2005) 3327.

[36] S. Haeberle, L. Naegele, R. Burger, R. Zengerle, J. Ducrée, Proc. 19th MEMS, Kobe, 2007, p. 497.

[37] S. Haeberle, L. Naegele, R. Burger, F. von Stetten, R. Zengerle, J. Ducrée, J. Microenc. $25(2008) 267$.

[38] J.A. Ko, H.J. Park, S.J. Hwang, J.B. Park, J.S. Lee, Int. J. Pharm. 249 (2002) 165.

[39] C.B. Patel, V.G. Gaikar, Sep. Sci. Technol. 39 (2004) 2655.

[40] Y.C. Shi, Y.M. Jiang, D.X. Sui, Y.L. Li, T. Chen, L. Ma, Z.T. Ding, J. Chromatogr. A. 742 (1996) 107.

[41] W.S.W. Ngah, C.S. Endud, R. Mayanar, React. Funct. Polym. 50 (2002) 181.

[42] P. He, S.S. Davis, L. Illum, Int. J. Pharm. 187 (1999) 53.

[43] L. Baruch, M. Machluf, Biopolymers 82 (2006) 570.

[44] K.D. Vorlop, J. Klein, Biotechnol. Lett. 3 (1981) 9.

[45] R. Bodmeier, O. Paeratakul, J. Pharm. Sci. 78 (1989) 964.

[46] E.E. Hassan, R.C. Parish, J.M. Gallo, Pharm. Res. 9 (1992) 390.

[47] C.H. Yang, K.S. Huang, J.Y. Chang, Biomed. Microdev. 9 (2007) 253.

[48] J.K. Hwang, H.H. Shin, Korea-Aust. Rheol. J. 12 (2000) 175.

[49] L.E. Nielsen, Polymer Rheology, Marcell Dekker, New York, 1977, p. 48. 


\section{Abbrevations}

APS: ammonium persulfate

Dex-HEMA: dextran-hydroxyethyl methacrylate

HA: hyaluronic acid

NIPAAm: $N$-isopropylacrylamide

PNIPAAm: $\operatorname{poly}(N$-isopropylacrylamide)

PAAm: poly(acrylamide)

PS: polystyrene

MeHA: methacrylated hyaluronic acid

PEG: poly(ethylene glycol)

PEGDA: poly(ethylene glycol) diacrylate

TPP: tripolyphosphate

WPI: whey protein isolate 
a) Micromolding [12]

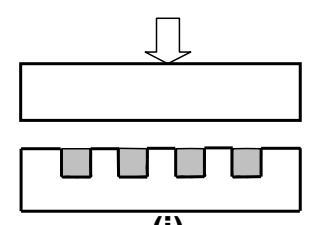

(i)

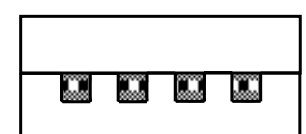

(iii)

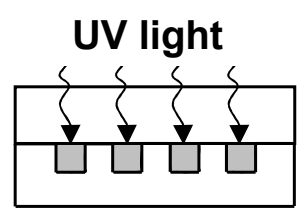

(ii)

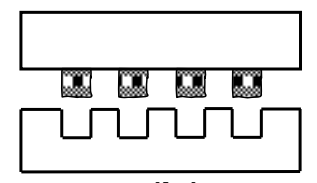

(iv) c) Microcapillary device [15]

b) Photolithography [13]

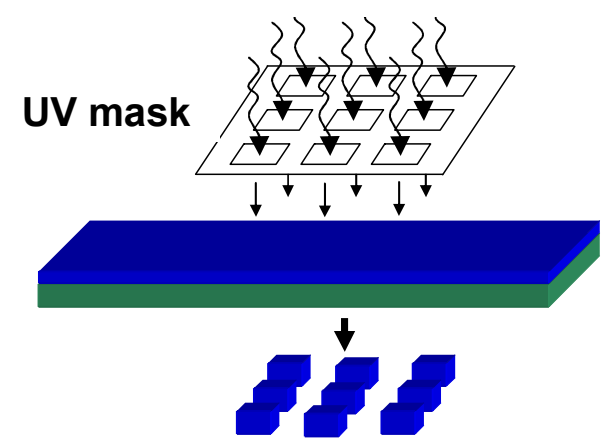

d) Sheath-flow microfluidic device [16]

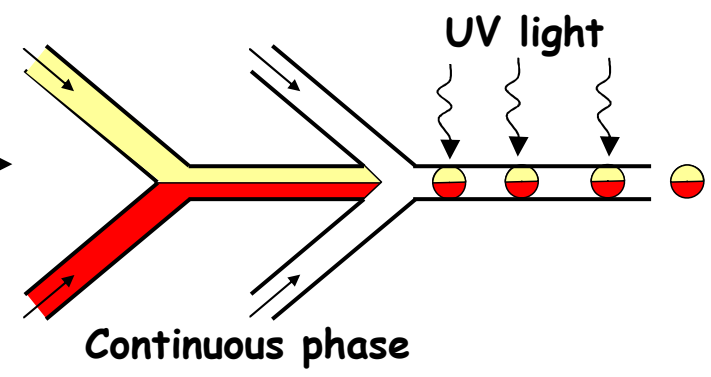

f) Membrane emulsification [18]

e) Microchannel emulsification [17]
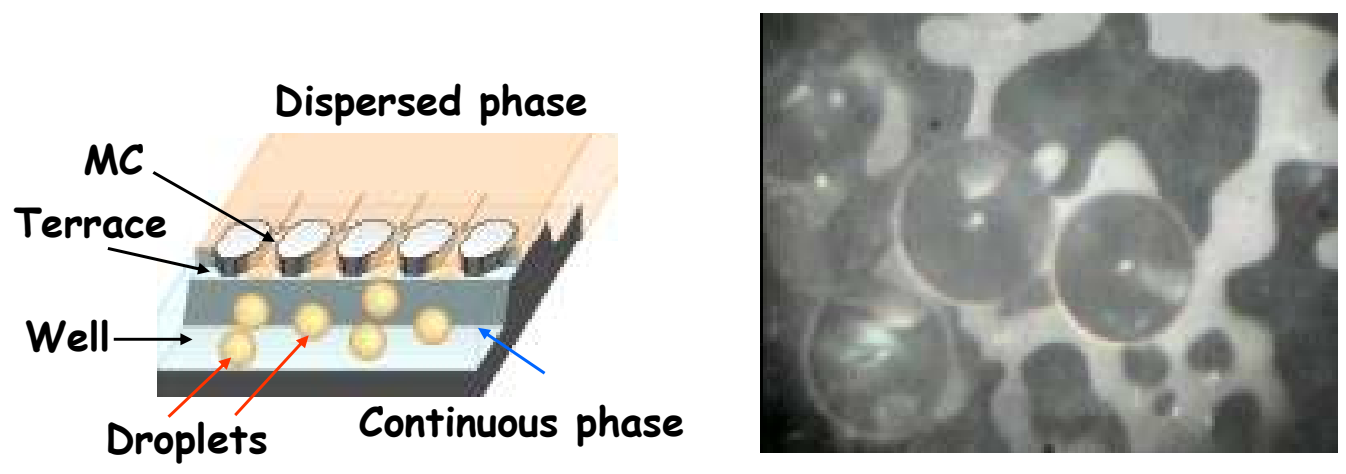

Figure 1. Innovative microengineering techniques for fabrication of hydrogel microbeads. 
a) Bifacial (Janus) gel
beads

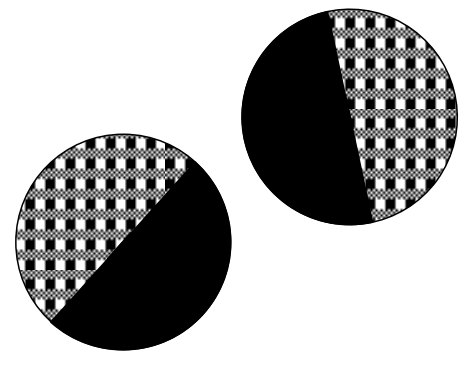
d) Gel beads with colloidal shells

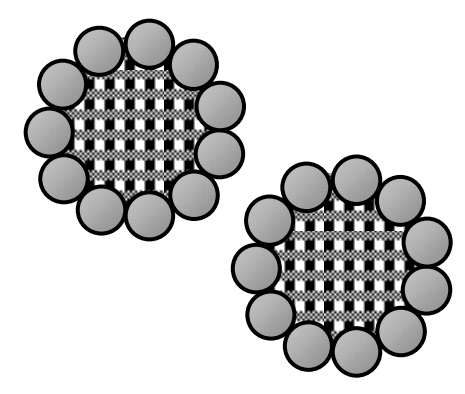

b) W/O/W emulsion drops with gelled inner droplets

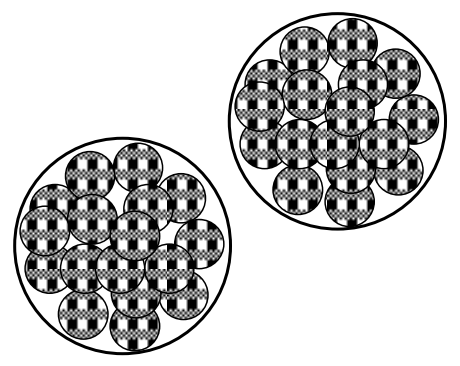

e) Discoid gel beads

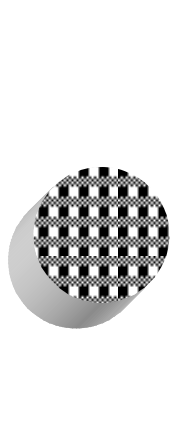

c) Hollow gel beads

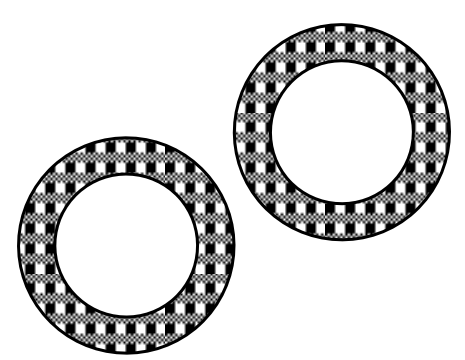

f) Square or hexagonal gel beads

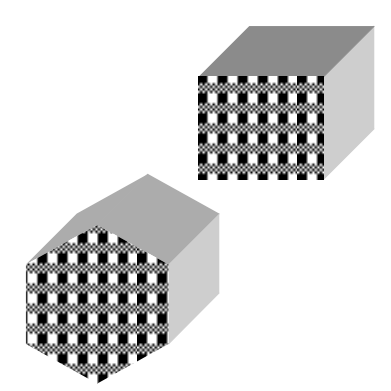

Figure 2. Examples of complex hydrogel microbeads fabricated through various microfluidic and membrane emulsification routes. 
a) Micromolding [20]

Alginate

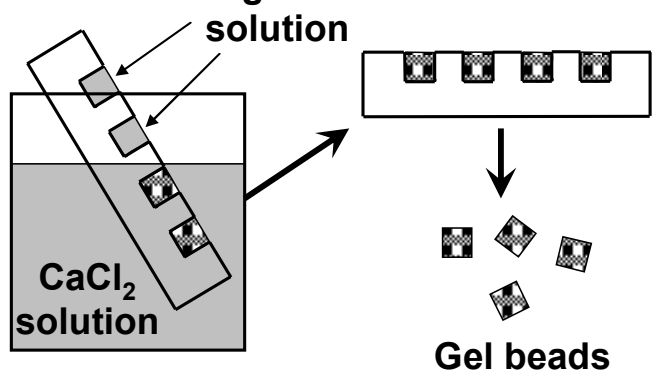

c) Air-flow nozzle [31]

Alginate solution

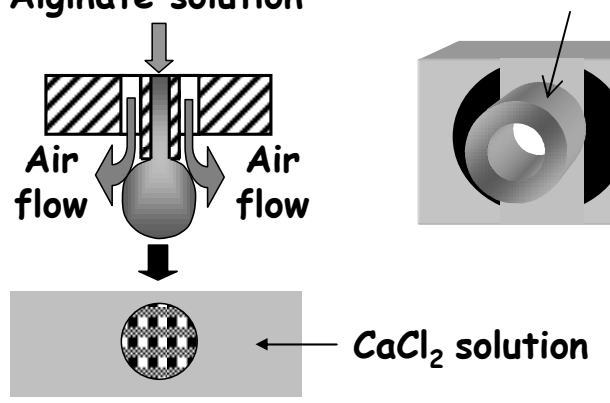

b) Direct mixing in $T$-junction

[30]

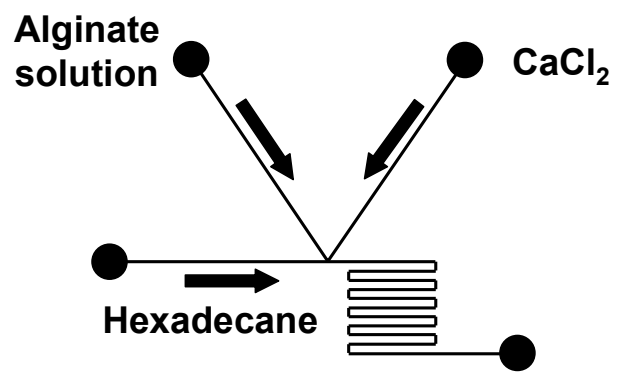

d) Multiple emulsion [32]

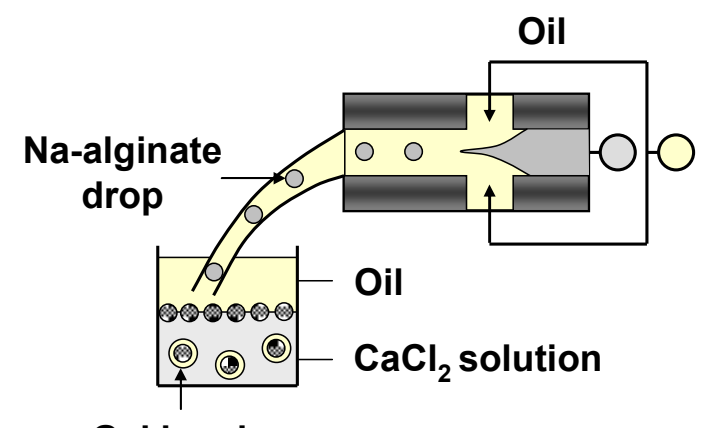

Gel beads

Figure 3. Microengineering techniques for production of calcium alginate microbeads by ionotropic gelation. The techniques are applicable for production of hydrogel beads of any biopolymer. 


\section{a) Internal gelation}

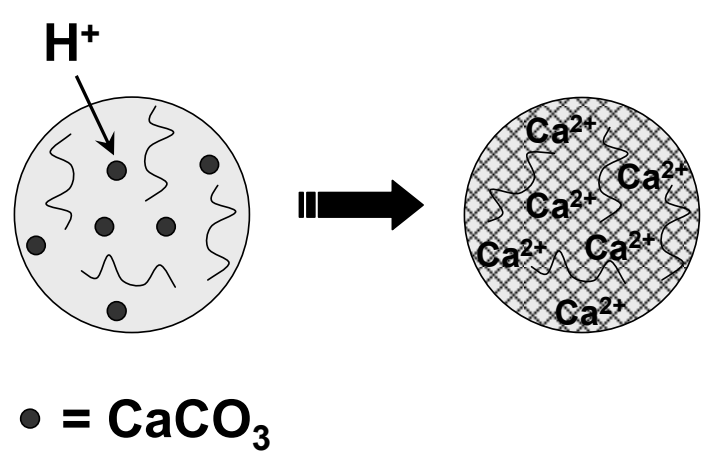

c) Coalescence-induced gelation

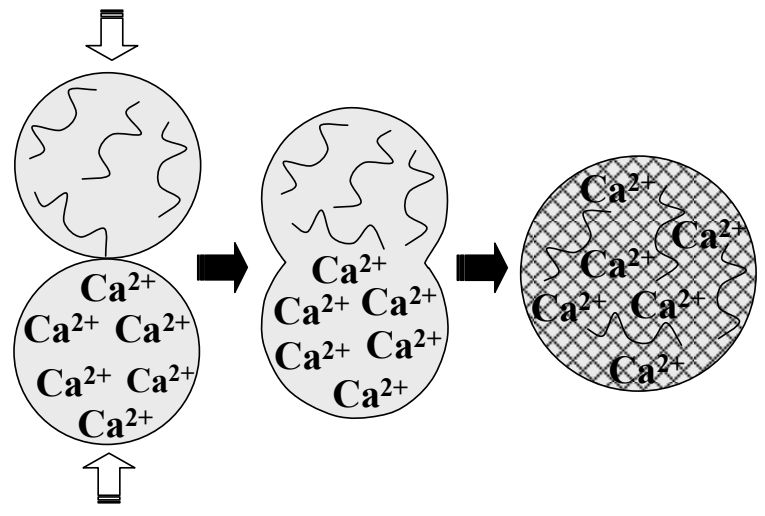

b) External gelation

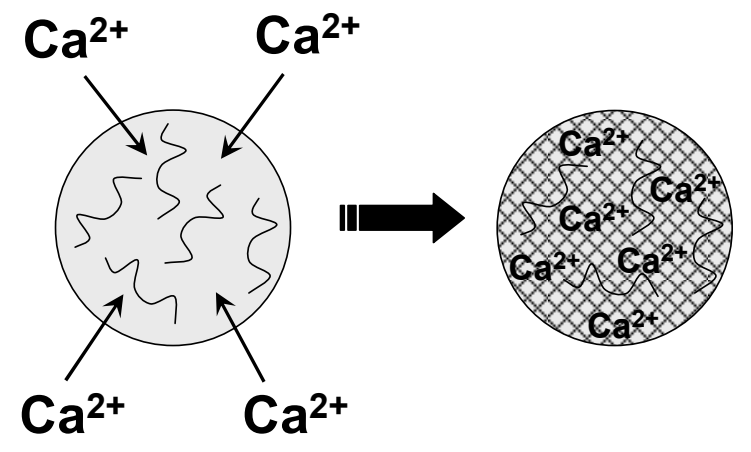

d) Rapid mixing in T-junction

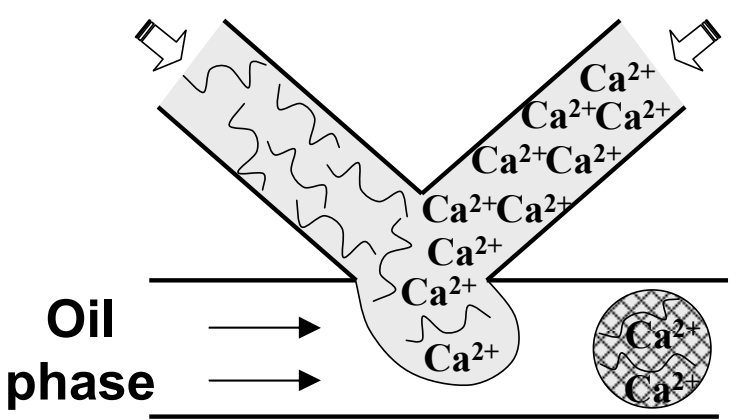

Figure 4. Main strategies for production of hydrogel beads by ionotropic gelation. For gelation of positively charged biopolymers, multivalent anions must be used instead of $\mathrm{Ca}^{2+}$. 


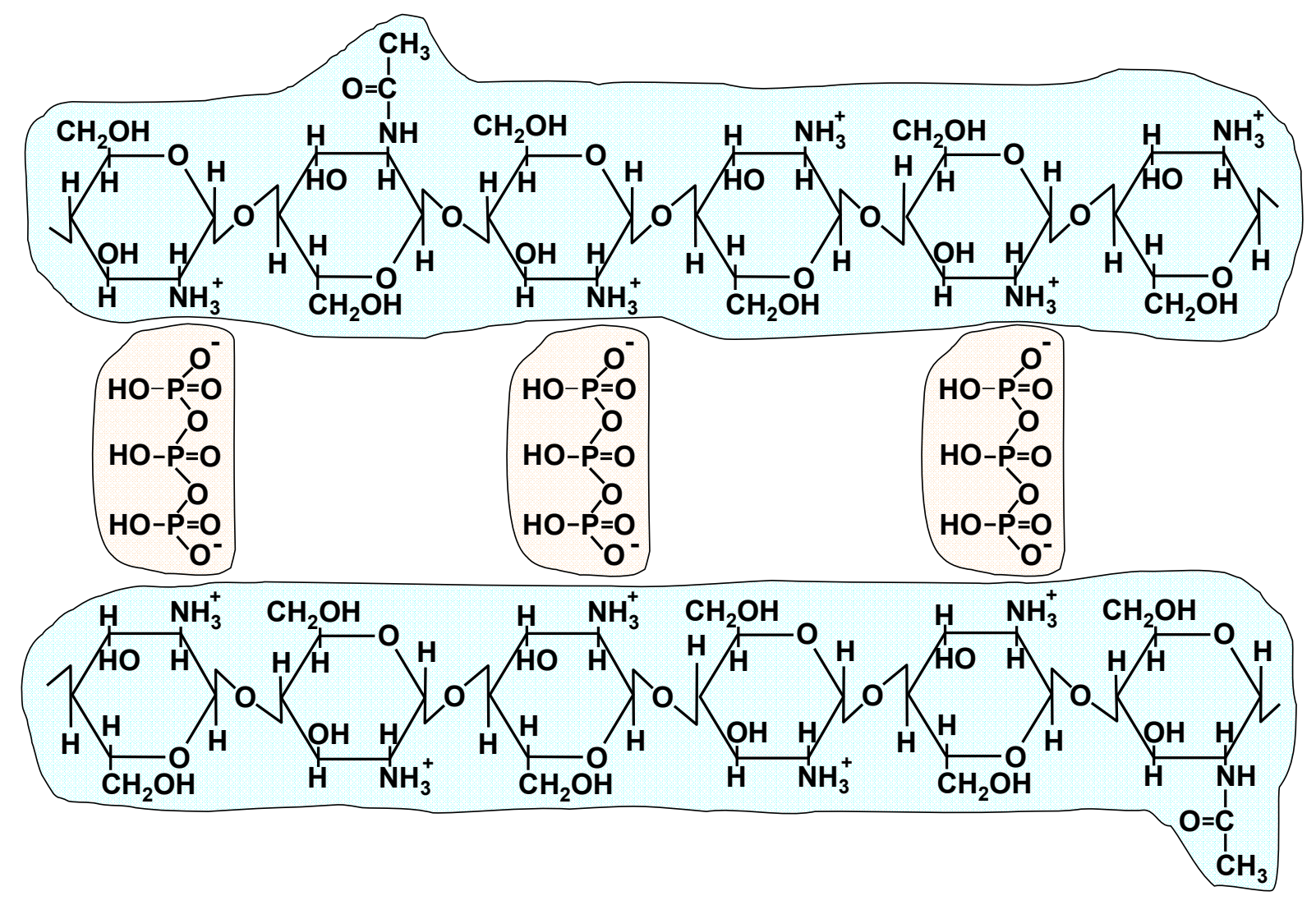

Figure 5. Structure of chitosan gel crosslinked with tripolyphosphate anions at $\mathrm{pH}$ below 6.5 . 


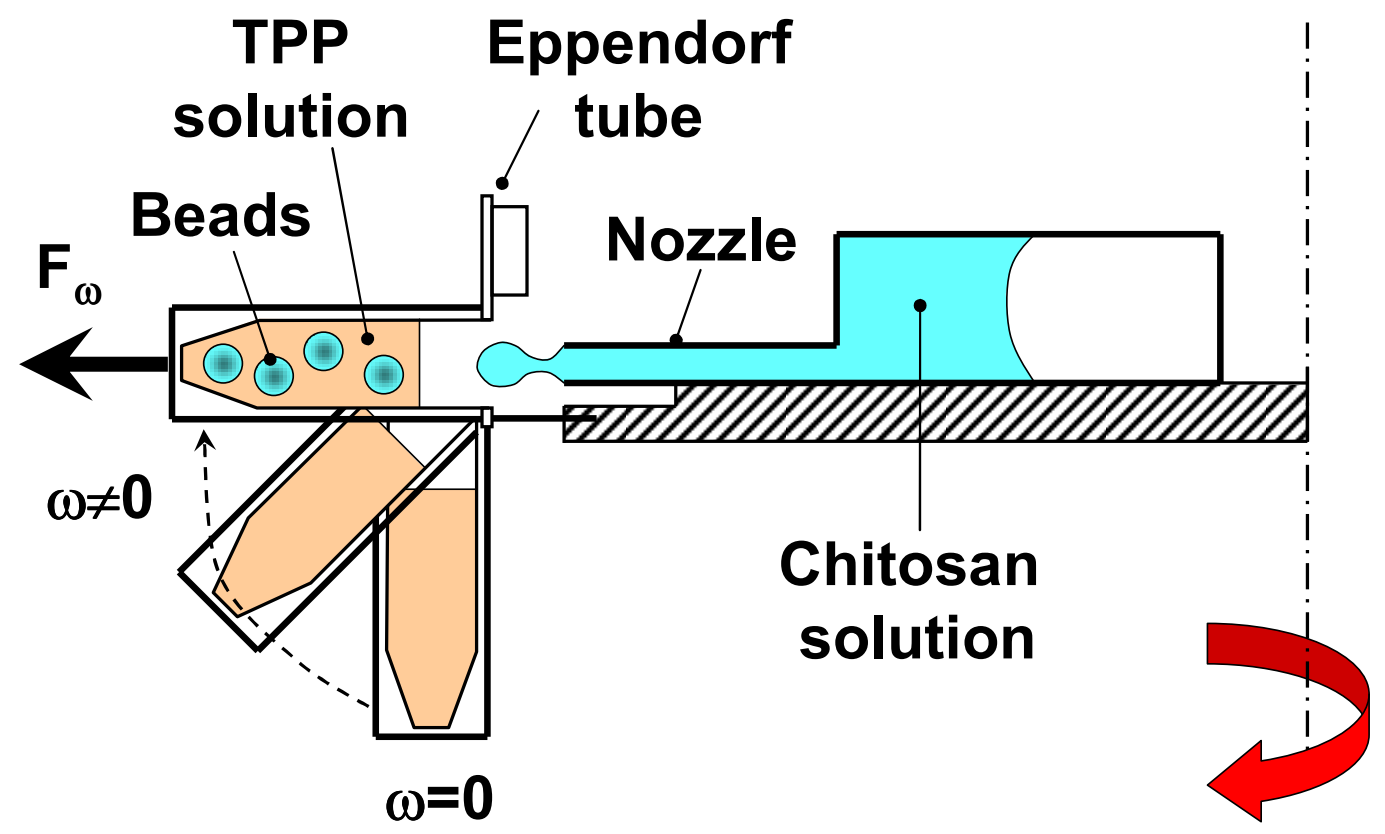

Figure 6. Schematic of the experimental setup consisting of centrifugal platform with a polymeric micronozzle and Eppendorf tube in a swinging bucket. 


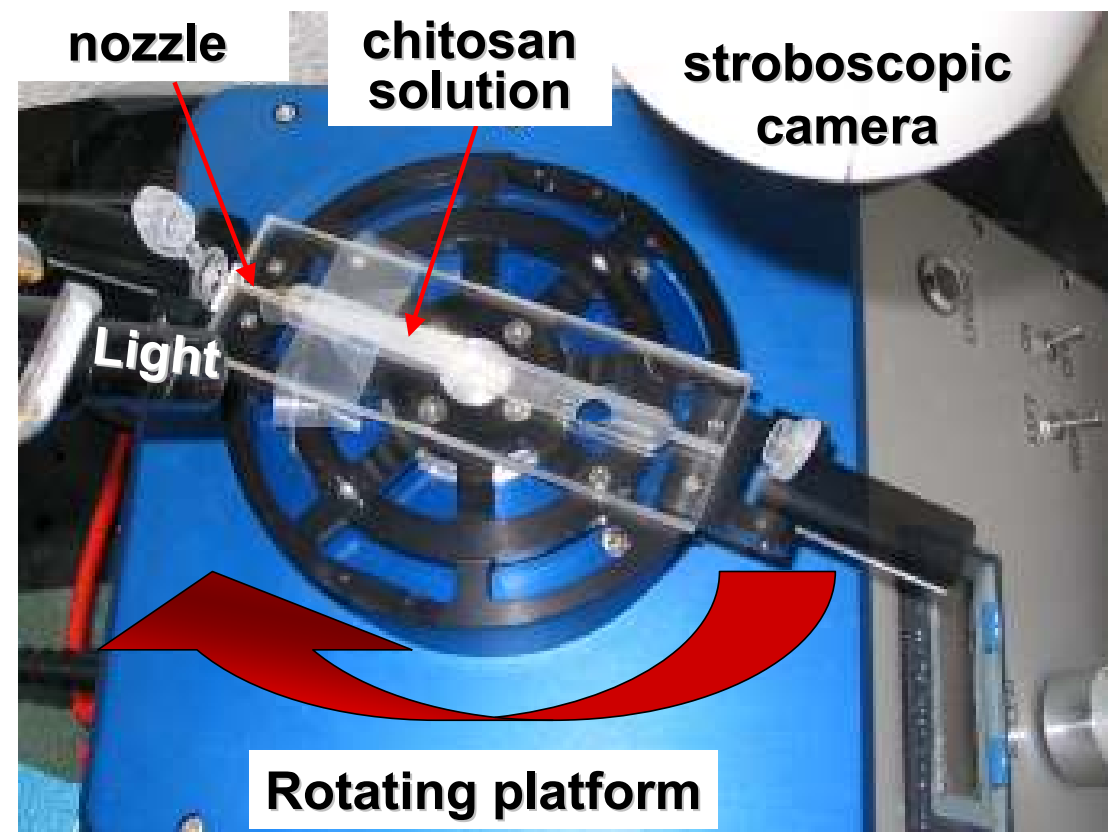

Figure 7. Photograph of the experimental set-up. 

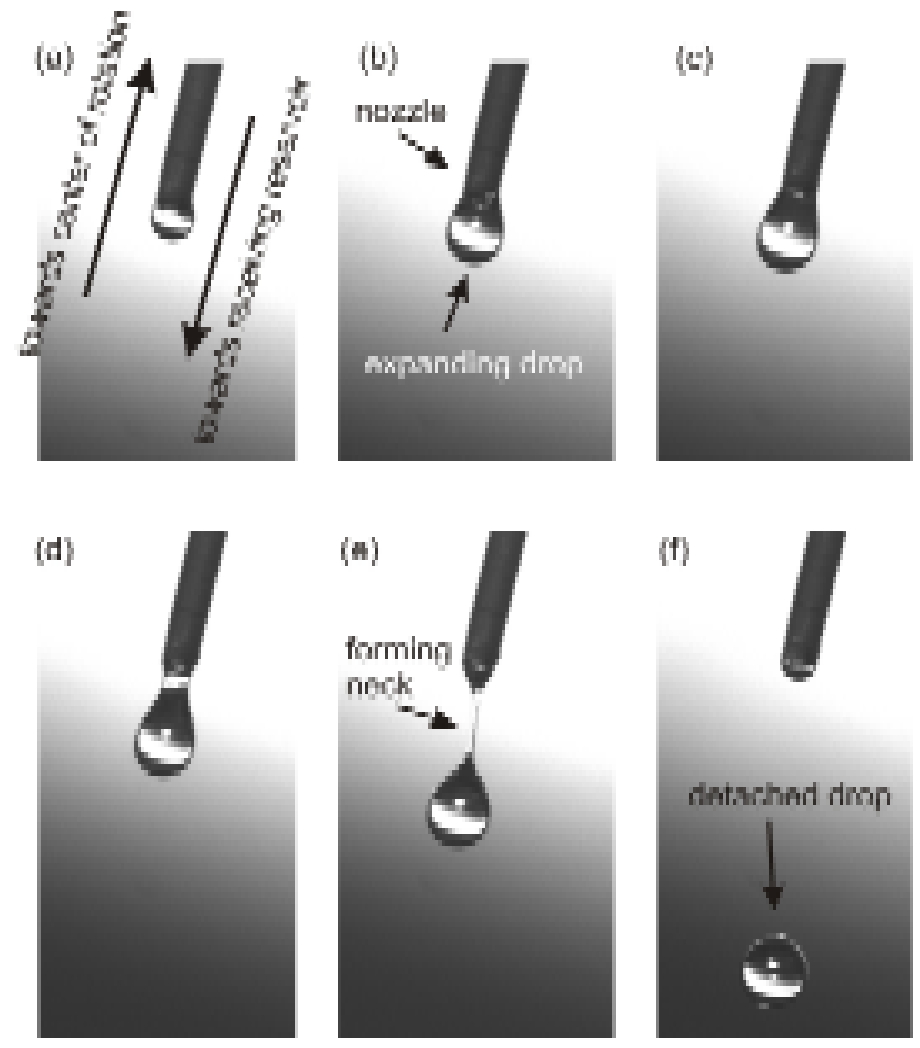

Figure 8. Sequential stroboscopic images of the droplet formation process at $25 \mathrm{~Hz}$ : (a)-(c) a liquid ( $2 \%$ chitosan solution) is subjected to a centrifugal pseudo-force field that leads to a droplet growth at the end of the nozzle. (d)-(e) The surface tension of the liquid favours a spherical shape which leads to a thinning neck between the droplet and the remaining liquid in the nozzle. (f) Finally, the droplet detaches and moves towards the receiving reservoir. 
(a)

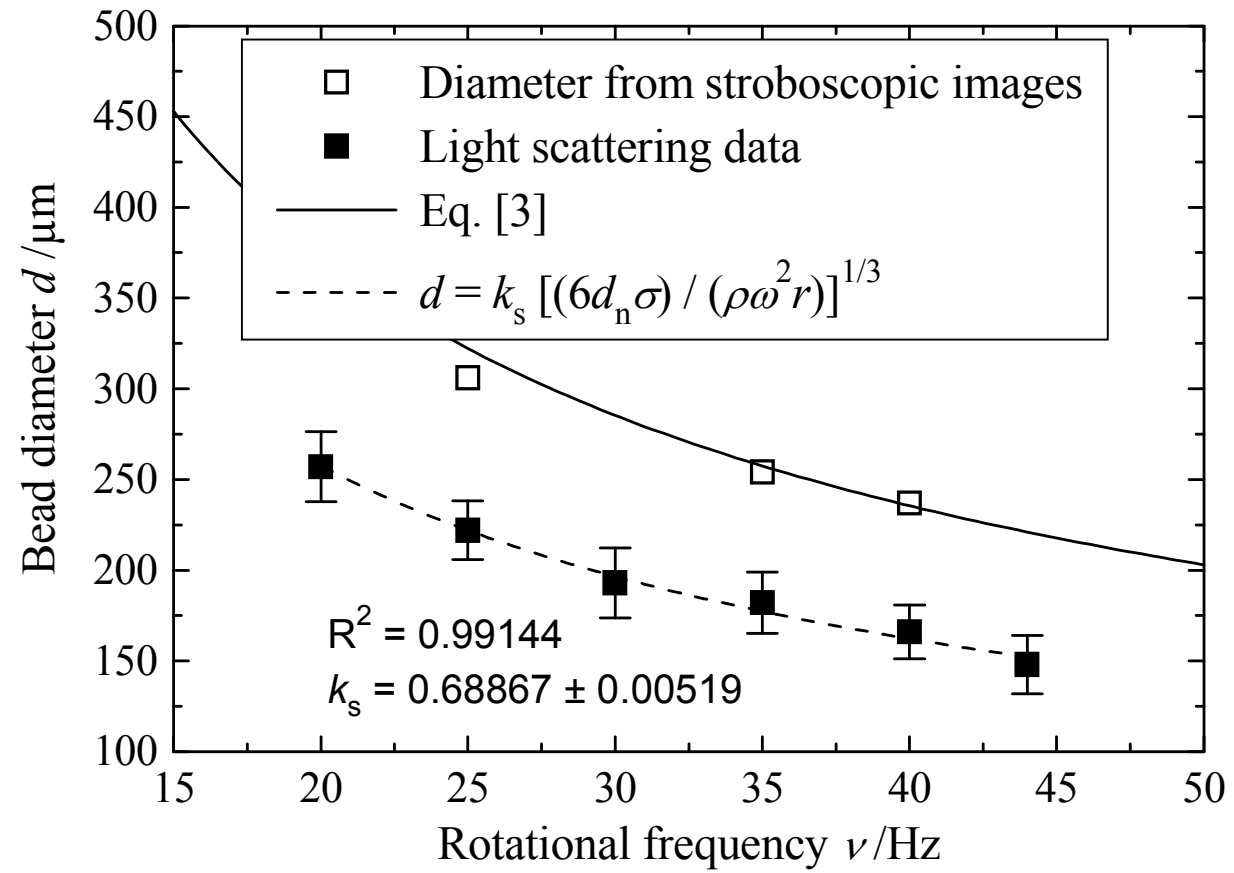

(b)

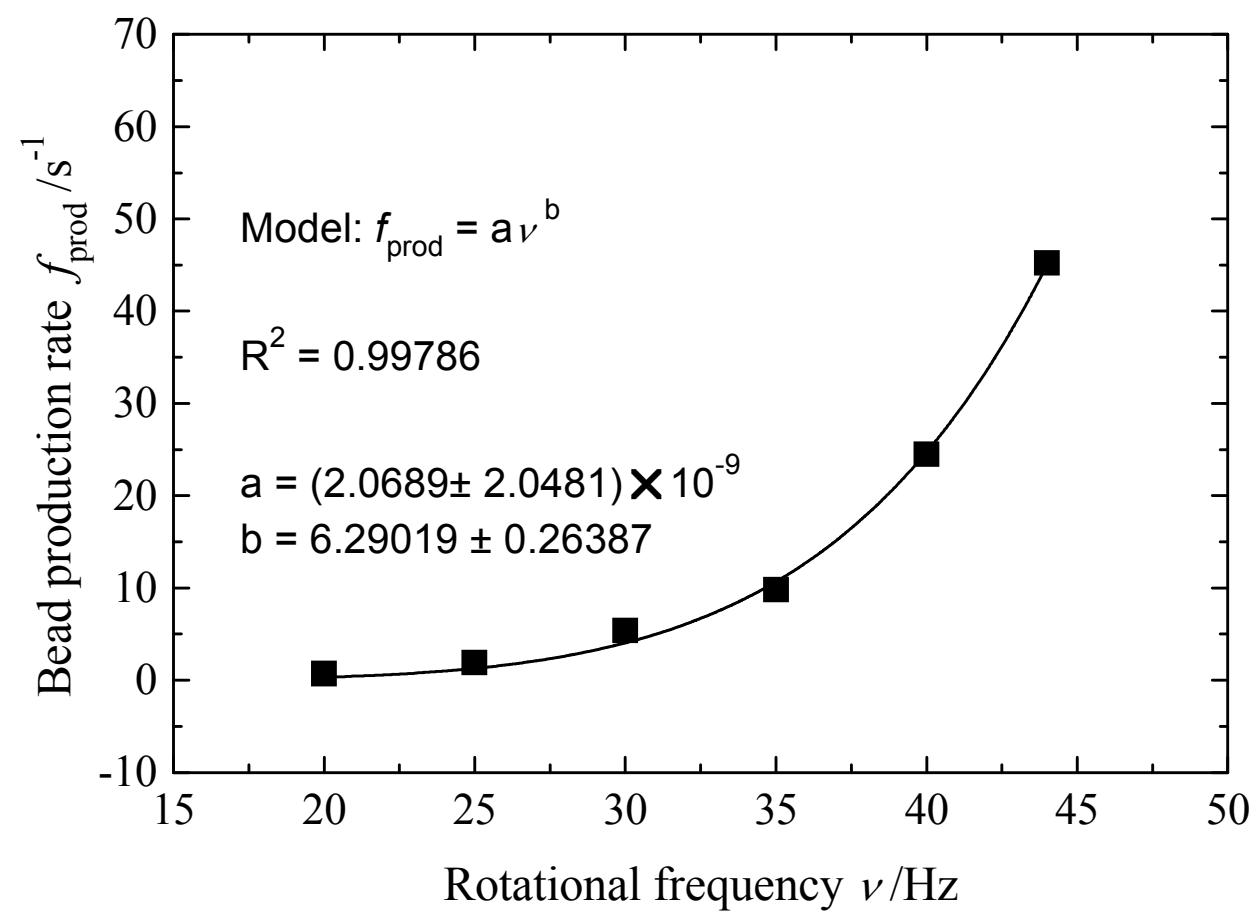

Figure 9. The characteristics of the bead formation: (a) diameters of hardened beads measured by light scattering compared to the diameters of the ejected drops observed on stroboscopic images. (b) bead production rates at different rotational frequencies. 
(a)

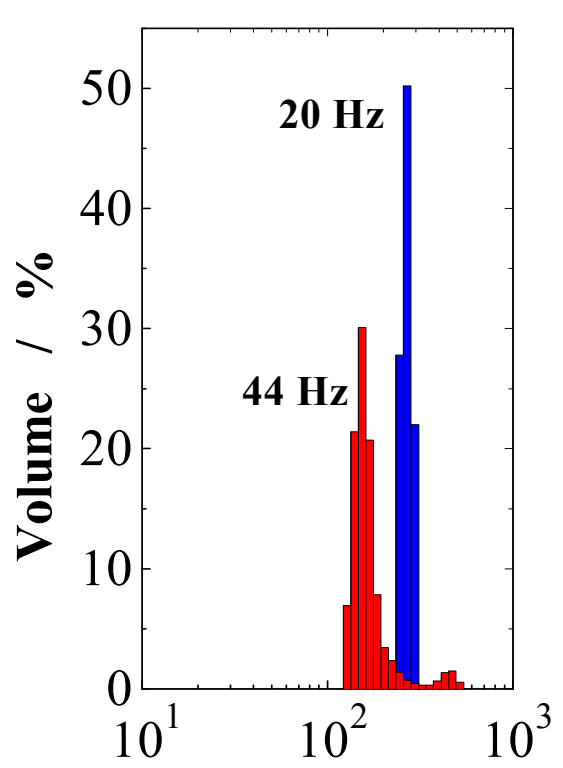

Particle size / $\mu \mathbf{m}$ (b)

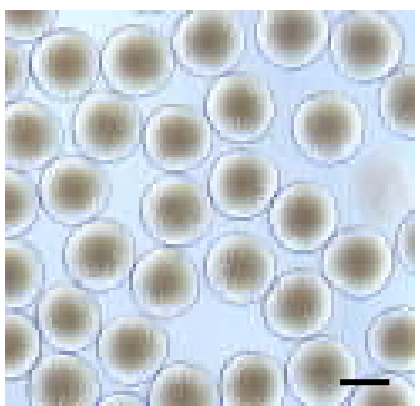

(c)

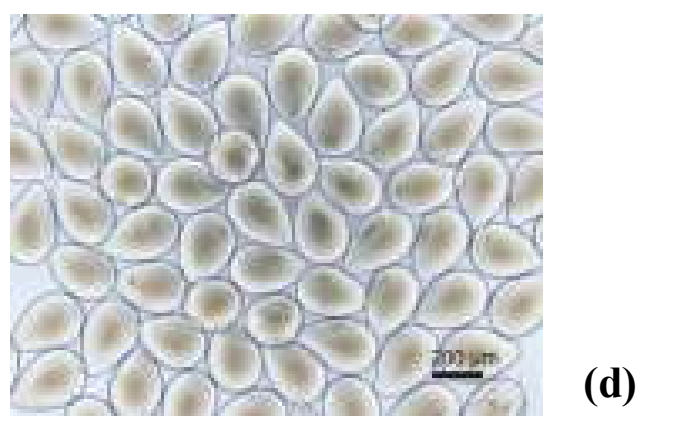

Figure 10 (a). Size distribution histograms of the hardened beads produced at 20 and $44 \mathrm{~Hz}$ with $2 \%$ chitosan. (b) Microscopic image of the beads produced at $20 \mathrm{~Hz}$ with $2 \%$ chitosan confirms a very uniform size and spherical shape without agglomeration (scale bar: $200 \mu \mathrm{m}$ ). (c) Beads produced at $25 \mathrm{~Hz}$ with $2 \%$ chitosan. The beads are smaller than at $20 \mathrm{~Hz}$, but retain its spherical shape. (d) Beads produced at $30 \mathrm{~Hz}$ with $3 \%$ chitosan. Due to the faster gelation and greater viscosity of the solution, the beads have a tear-like shape, because the flying drops rapidly gelify when they come into contact with the hardening solution. 